\title{
Analysis and prediction of the summer cooling load characteristics
}

\author{
XU Hao ${ }^{1}$, CHEN Lipin ${ }^{1}$,YE Qiang ${ }^{1}$, REN ZhiChao ${ }^{1}$, LIANG Yuan ${ }^{2}$ \\ ${ }^{1}$ State Grid Sichuan Economic Research Institute SGSERI Chengdu, China \\ ${ }^{2}$ College of Management Science, Chengdu University of Technology \\ Chengdu, China
}

\begin{abstract}
Nowadays, cooling load is mainly attributable to upgrade of a new high of power grid load. An accurate calculation and prediction of cooling load can better meet power demands at peak hours in summer. Based on the historical summer cooling load and temperature data of Sichuan province, the characteristics of summer cooling load and temperature in Sichuan are examined firstly. Next, the random and nonlinear relations of power load are studied. Finally, the BP neural network model optimized by the genetic algorithm (GA) is employed to achieve accurate prediction of summer cooling load in Sichuan province. Results suggest that cooling load is closely connected with temperature and air-conditioner ownership, and that the BP neural network optimized by the GA is effective in predicting summer cooling load. To sum up, this research can provide solid basis for power load scheduling.
\end{abstract}

\section{Introduction}

Cooling load refers to rapid increase of the power grid network within a short period of time due to launch of cooling equipment, notably the air-conditioner [1]. In recent years, along with rapid development of economy, continuous improvement of people's living standards, and worsening of the "heat island" phenomenon, cooling load is increasing on an annual basis [2][3]. Nowadays, cooling load has been a major factor attributable to continuous upgrade of a new high of the power grid load in summer. This has not only changed the original load characteristics [4-6], but also brought negative effects on the system voltage and frequency stability [7].

The special landform of the Sichuan Basin has led to a moist and scorching hot summer in most parts of Sichuan. To cool down the temperature, Sichuan has a high requirement of cooling load in summer, which has a significant influence on power load. In order to increase accuracy of load prediction at different time scales and to ensure stability, safety and economical operation of Sichuan's power grid in summer, it is imperative to measure and analyze cooling load of Sichuan's power grid.

Calculation of cooling load should take into account not only the local climate conditions, but also the local use habits of cooling equipment (e.g. air-conditioners), national energy-saving policies [8], and perception of physical comfort [9]. Since it is no easy task to collect all the necessary data, accuracy of cooling load calculation has long remained a challenge. Traditional algorithms for power load prediction include time series, multiple linear regression and Fourier component. Every algorithm, with respective advantages and disadvantages, is applicable to different occasions. Since power load changes along with weather conditions and human activities, a series of random and nonlinear relations has formed, making it hard to improve the power load prediction accuracy [10]. As a universal approximator, the artificial neural network can recognize systems, which are nonlinear in essence. Therefore, the artificial neural network can be regarded as a highly effective load prediction technology. Characterized by large-scale parallel, distributed storage and processing, selforganization, self-adaption and self-learning ability, the neural network is especially suitable for inaccurate, fuzzy information processing with many factors and conditions to take into consider.

In optimizing the back propagation (BP) neural network, the genetic algorithm (GA) can improve efficiency and robustness of global searching, and has shown outstanding performance in practical application. After the neural network is globally optimized by the GA, the BP algorithm can be adopted for fine tuning to accelerate the network convergence speed and reduce the probability of training failure [12].

This paper collects and processes the power load and temperature data in June, July and August of 2009 to 2016. Then, statistical analysis is adopted to sum up rules and influencing factors of power load changes. General changes of power load are roughly predicted. Finally, the BP neural network model optimized via the GA is employed to achieve precise prediction of cooling. It is hoped that this research can contribute to contribute to the power load prediction and scheduling in Sichuan province. 


\section{Analysis of summer cooling load characteristics in Sichuan province}

The power load and temperature historical data of Sichuan province in June, July and August from 2009 to 2016 are collected, analyzed, and used for load prediction. Results show October and April are the two months with the most comfortable temperature in Sichuan in that almost no cooling equipment is required. Hence, the mean value of power grid in October and April is adopted as a reference load for calculation of cooling load. The maximum daily cooling load is calculated as:

$\Delta \mathrm{L}=$ Daily maximum power load-( Daily maximum power load in October+ Daily maximum power load in April)/2

\subsection{Consistency between daily maximum cooling load and daily highest temperature changes}

As shown in Fig. 1 and Fig. 2, the daily maximum cooling load changes via the daily highest temperature, and the change trend of the two is consistent with each other. To be specific, with increase of the daily highest temperature, the daily maximum cooling load also rises. On the contrary, when the daily highest temperature decreases, the daily maximum cooling falls.

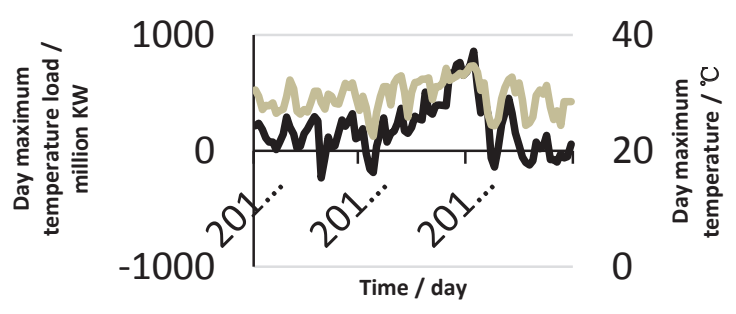

Figure 1. Change trend of the daily maximum cooling load and the daily highest temperature from June to August 2015

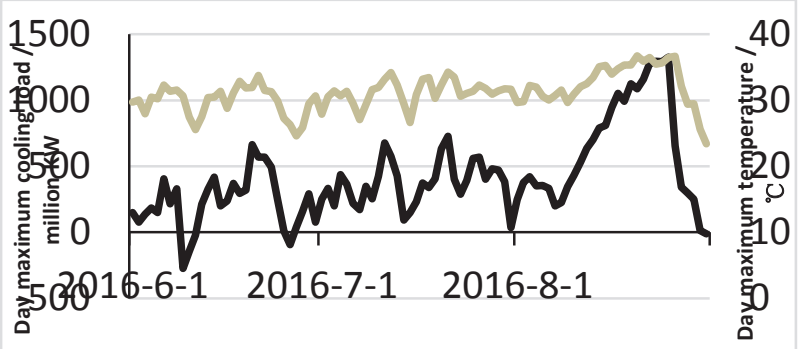

Time / day

Figure 2. Change trend of the daily maximum cooling load and the daily highest temperature from June to August 2016

The daily highest temperature has an accumulative effect on the daily maximum cooling load. From Fig. 1 and Fig. 2, it can be seen that, after days of high temperature above $30{ }^{\circ} \mathrm{C}$, the power load increases rapidly, because the human body has an upper limit for continuous high temperature. (The dark broken line below represents the load; while the light broken line above represents the temperature.)

\subsection{Influence of GDP on the daily maximum cooling load long-term change trend}

As one notices in Fig. 3, the daily maximum cooling load of Sichuan's power grid experiences continuous growth from 2009 to 2009, and the percentage of the maximum cooling load in the daily load is also on an upward trend.

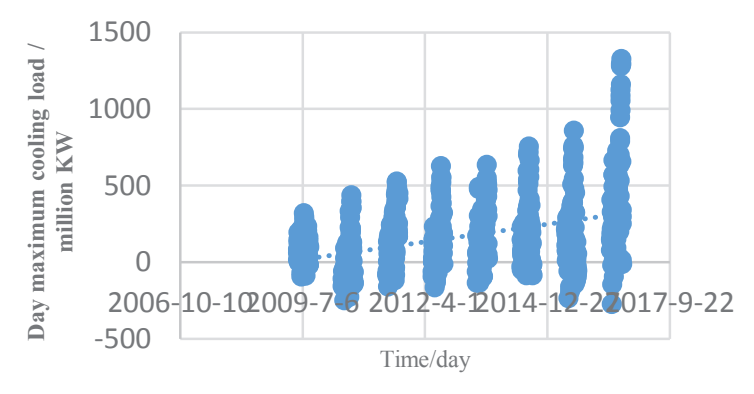

Figure 3. Percentage and linear change trend of the daily maximum cooling load from June to August of 2009 to 2016

As one observes in Fig. 4, the annual summer daily highest temperature shows no dramatic changes from 2009 to 2016. This provides solid basis for the conclusion that the daily maximum cooling load is positively correlated with the daily highest temperature. The daily maximum cooling load and the daily highest temperature changes represent changes in the short term. The annual changes of the daily maximum cooling load are changes in the long term, and are affected by the annual GDP of Sichuan province. The change trend of the GDP is shown in Fig. 5. (Unit: 100 million yuan) It is widely agreed that growth of GDP can contribute to improvement of people's living standards. With basic living demands satiated, people pay more attention to comfortability of their living and production environment. To the end, quantity and use frequency of cooling equipment both increase, thus leading to rise of the daily maximum cooling load.

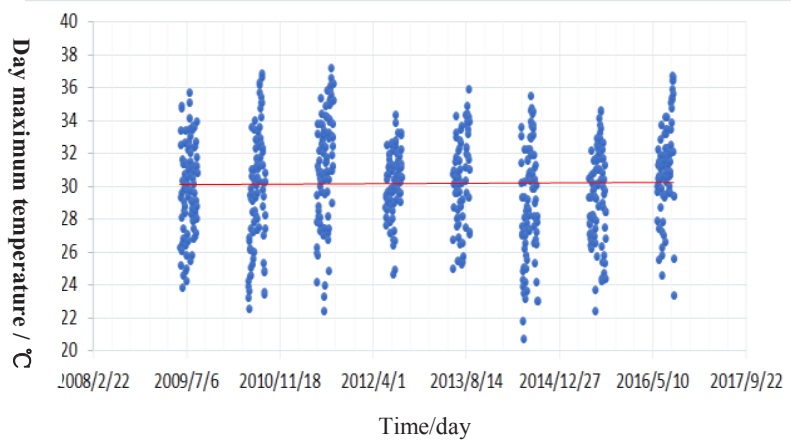

Figure 4. Changes and linear change trend of the daily highest temperature from June to August of 2009 to 2016 


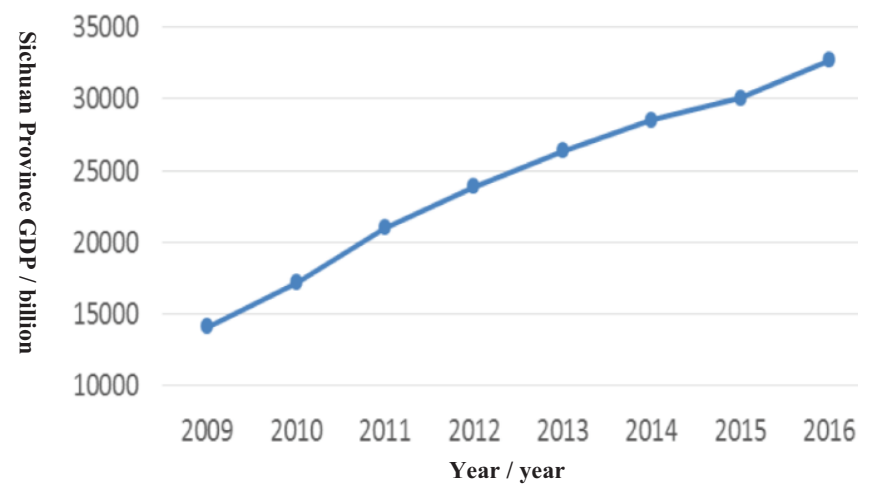

Figure 5. Sichuan provincial GDP level from 2009 to 2016

\subsection{Influence of small hydropower stations on the daily maximum cooling load}

A negative maximum cooling load is found in each year from 2009 to 2016 . When the maximum cooling load is negative, it suggests the total load of the current day is lower than the reference load of the current year. One possible reason for the phenomenon is that there might be other forms of energy supply or that the load required by production activities decreases.

Through statistical analysis of annual data, it can be found that the maximum cooling load concentrates in July, seldom appearing in June and August. Historical data show that July is a month with abundant precipitation. Besides, there are many small hydropower stations in Sichuan, which serve as an important supplementation to the national power grid. Therefore, though data of small hydropower stations are incomplete, this paper still believes that the negative maximum cooling load results from ample power supply from small hydropower stations during the period of abundant precipitation period. On the Dragon Boat Day, the maximum cooling load is mostly negative. This represents a typical decrease of load required by production activities.

\section{Summer cooling load prediction}

\subsection{Prediction model and algorithm}

In this paper, the BP neural network model optimized via the genetic algorithm (GA) is adopted for prediction of summer cooling load in Sichuan province. The GA is an algorithm for global optimization and random search. On the basis of individual gene representative, the genetic operator is adopted to simulate various phenomena occurring during the process of inheritance, including replication, intersection and variation, and pick the superior individuals from the population. Finally, the optimal individuals can be obtained. In this paper, the improved GA and BP neural network are combined to form a BP neural network prediction model optimized by the GA algorithm.

The model consists of the following three parts:
(1) Determining the BP neural network structure: The BP neural network topological structure is determined according to the chaotic time series input and output of parameters. Based on that, the length of the GA individuals can be determined.

(2) Optimizing the BP neural network weight and threshold value using the GA: The linear interpolation function is adopted to generate the population individuals. Every individual in the population contains a BP neural network weight and threshold value. The degree of individual fitness is calculated using the fitness function. The GA search the corresponding individual with the optimal fitness value via a series of operations, including selection, intersection and variation.

(3) Predicting the BP neural network: The optimal individual obtained via the GA is used to assign the initial weight and threshold value of the BP neural network. Then, the BP neural network is adopted to conduct local optimumseeking so as to obtain the predicted value with the global optimal solution.

Below are steps of the model:

Step 1: Set the population size as p;

Step 2: Confirm the individual evaluation function;

Step 3: Use the roulette method to choose the operator; (Select the chromosome from every generation of population based on the percentage of the fitness degree.)

Step 4: Adopt the real number cross method considering the real number coding of individuals;

Step 5: Choose the $\mathrm{j}$ gene from the $\mathrm{i}$ individual for variation;

Step 6: Decompose the optimal individual obtained by the GA into the connecting weight and threshold value of the BP neural network;

Step 7: Use the BP algorithm to train the BP neural network prediction model and work out the optimal solution of the chaotic time series prediction.

Here, the feedforward neural network (FNN), empirical mode decomposition (EMD), support vehicle machine (SVM), BP neural network and GA-optimized BP neural network are used for prediction. Their prediction performance is compared. Due to limit of the paragraph, the model theory is not further expounded.

\subsection{Prediction results and performance}

The daily maximum cooling load and daily highest temperature historical data from June to August of 2009 to 2016 are adopted as research data. There are $604 * 7$ sample data in total, and 92 data in 2016 are predicted. It is found out that the variation between the predicted value and the actual value is small when the GAoptimized BP neural network is adopted for prediction. Indexes to verify the prediction results include MSE, 
MAE, rMAE, MAPE and RMSPE, and the index data are presented in Table 1.

Table 1. Evaluation indexes of prediction performance

\begin{tabular}{|c|c|c|c|c|c|}
\hline & MSE & MAE & rMAE & MAPE & RMSPE \\
\hline FNN & 31501 & 135.7970 & 0.3649 & 1.7608 & 6.4412 \\
\hline EMD_FNN & 121340 & 256.9908 & 0.6578 & 5.8897 & 46.5189 \\
\hline SVM & 171630 & 1495.2 & 0.0404 & 4.1540 & 1.1767 \\
\hline BP neural network & 1344.1 & 12.7733 & 0.0316 & 0.0226 & 0.0514 \\
\hline $\begin{array}{c}\text { Genetic algorithm optimization } \\
\text { BP neural network }\end{array}$ & $\mathbf{\underline { \mathbf { 1 6 . 8 6 0 6 } }}$ & $\mathbf{\mathbf { 1 . 4 7 4 7 }}$ & $\mathbf{0 . 0 0 3 6}$ & $\mathbf{0 . 0 0 4 2}$ & $\mathbf{0 . 0 0 7 7}$ \\
\hline
\end{tabular}

According to the index data and evaluation criteria in Table 1, it is obvious that the GA-optimized BP neural network model is the best, and can meet the prediction requirements.

\section{Conclusions}

Through descriptive statistics of the daily maximum cooling load and daily highest temperature data, the following conclusions can be reached:

(1) The change trend of the daily maximum cooling load and the daily highest temperature generally coincides with each other, but the latter has a cumulative effect on the former;

(2) Temperature is a major factor influencing the daily maximum cooling load in the short term, while GDP is a long-term influencing factor of the daily maximum cooling load;

(3) A major cause of the negative daily cooling load is the large number of small hydropower stations in the period of abundant precipitation.

Analysis of the daily maximum cooling load prediction results and performance shows that the GAoptimized BP neural network model outperforms other prediction models in terms of every prediction index. Therefore, the GA-optimized BP neural network algorithm can be combined with the local weather forecasts to help predict the future maximum cooling load in June, July and August of Sichuan province.

\section{References}

1. Yao Wei, Cheng Shijie, et al. Application of HVDC technology in grid - connected offshore wind farm CEP, 40 (2007)

2. Liu Lin, Ge $\mathrm{Xu}$ Bo, and so on. China 's offshore wind power development status and analysis.ETE, 24(2012)

3. Wang Jingquan, Cheng Jiansheng, Li Feng. On the construction of offshore wind farms in China .CES, 12(2010)

4. Yin Yufen. Influence of temperature on regional load characteristics. GEPr, 24(2011)

5. Li Canbing, Shang Jincheng, Zhu Shouzhen, and so on. Analysis of Energy Consumption Caused by
Cumulative Effect of Temperature on Temperature Regulation Load. Power System Automation, 34 (2010): 30-33.

6. KYUNG B S, SEONG K H, JUNG W P, et a1. Hybrid load forecasting method with analysis of temperature sensitivities. IEEE Trans Oil Power Systems, 21(2006): 869-876.

7. Zhang Limin, $\mathrm{Fu}$ Hongjun, $\mathrm{Li}$ Yuyan, et al. Estimation of Cooling Load Ratio of Air Conditioning and Its Influence on Voltage Stability. Relays, 33 (2005): 33-36.

8. TONG Shulin, WEN Fushuan. Calculation and analysis of the annual maximum high-temperature related load in the energy saving and emission reduction environment in Guangdong Province.Journal of North China Electric Power University, 37(2010):32-37.

9. TAO Yong, SHEN Ying. Influence of summer weather condition on regional air conditioning loads . East ChinaElectric Power, 34(2006):29-30.

10. Moazzami M, Khodabakhshian A. Hooshmand R. A new hybrid day-ahead peak load forecasting method for Iran's National Grid. Appl Energy 101(2013):489-501.

11. Lawan S, Abidin W, Masri T, Chai W, Baharun A. Wind power generation via ground wind station and topographical feedforward neural network (T-FFNN) model for small-scale applications. Journal Of Cleaner Production143(2017) :1246-1259.

12. WANG Jun-tao.Study on Optimized BP Neural Network Algorithm Based on Genetic Algorithm. Small and Medium Enterprises Management and Technology, 4 (2017).

13. Wang J, Shang L, Chen S, et al. Application of fuzzy classification by evolutionary neural network in incipient fault detection of power transformer.IEEE International Joint Conference on Neural Networks, (2004)

14. Han Liqun. Artificial neural network theory, design and application. Beijing. Chemical Industry Press .(2007)

15. Huang N E, Shen Z, Long S R, et al. The Empirical Mode Decomposition and the Hilbert Spectrum for Nonlinear and Non-Stationary Time Series Analysis. Proceedings Mathematical Physical \& Engineering Sciences, 454 (1998):903-995.

16. Vapnik V N. The nature of statistical learning theory. New York Springer, (1995).

17. Mukherjee S, Osuna E, Girosi F. Nonlinear prediction of chaotic time series using support vector machines. Proceedings of IEEE NNSP,(1997)

18. Yan Pingfan, Zhang Changshui. Artificial neural network and simulated evolutionary computation. Beijing: Tsinghua University Press, (2005) 